\title{
Investigating Students’ Ideas About X-rays While Developing Teaching Materials for a Medical Physics Course*
}

\author{
Spartak Kalita and Dean Zollman
}

Department of Physics, Kansas State University, Manhattan, KS, 66506-2601

\begin{abstract}
The goal of the Modern Miracle Medical Machines project is to promote pre-med students' interest in physics by using the context of contemporary medical imaging. The X-ray medical imaging learning module will be a central part of this effort. To investigate students' transfer of learning in this context we have conducted a series of clinical and teaching interviews. In the latter interview, some of the proposed learning materials were used. The students brought to our discussion pieces of knowledge transferred from very different sources such as their own X-ray experiences, previous learning and the mass media. This transfer seems to result in more or less firm mental models which often are not always internally consistent or coherent.
\end{abstract}

Keywords: physics education, medical physics, transfer of learning, mental models, X-rays

\section{INTRODUCTION}

Pre-med students often complain that physics classes lack relevance to their future profession and the traditional required physics curriculum rarely makes any effort to relate the content to this vast (and mainly diligent) student population. Some of these issues have been addressed in Medical Physics courses designed and implemented around the country in recent years, [1,2] but these are optional courses that are designed to follow compulsory physics classes and do not replace them. Thus, a more systematic effort here is urgent.

For this purpose the Modern Miracle Medical Machines (MMMM) project has been undertaken. Its main goal is to conduct research on the reasoning and models that students use as they transfer basic physics knowledge in the application of physics to contemporary medicine. [3]

We utilize the general framework for dynamic transfer of learning that was developed by the KSU Physics Education Research Group [4]. We seek evidence of transfer from physics and other science classes, students' personal experiences and any other sources that students may find relevant.

The X-ray module will be a central one in our MMMM instruction. However, we have not found in the literature any explorations of students' understanding of X-rays. This lack of effort can be easily explained - the concept of X-rays is not so universal and omnipresent in our life (as, for instance, light that is somewhat connected to it) and probably would not require our special attention if it were not for the purpose of making the algebra-based physics course more appealing and relevant to pre-med students.

For other proposed modules in the MMMM project, such as Positron Emission Tomography or Magnetic Resonance Imaging, the investigation of students' pre-conceptions probably would not be useful as these topics are not covered in regular courses even in passing and students know them in the best case only by their names.

For the purpose of the development of the X-rays module our situation is fortunately a bit better. Almost all of the students either have undergone some X-ray procedure in their lives or know of someone who has. They already have some preconceived ideas about how it might work or may be strongly inclined to build models right on the spot when asked to do it even if they haven't thought about it before (as was confirmed by our research).

\footnotetext{
* Supported by NSF grant \# 04-26745
} 


\section{METHODOLOGY}

\section{Phase 1 - Fall 2004}

At the very beginning, a series of preliminary unstructured interviews (with some semi-structured elements modeled after Piaget [6]) was conducted. The protocol format allowed follow-up questions and thus questions that come later in the prepared list were able to be modified or even omitted if a student had already answered them in one of the follow-up series of questions.

In this preliminary study we had not yet narrowed our attention on the pre-med student population and we were looking at students' ideas about $\mathrm{X}$-rays in general. Thus, we decided to interview students with various backgrounds and very different levels of preparation. Among 16 of these students eight were from a conceptual physics class - 4 females studying elementary education and 4 males who were nonscience majors. 8 were from a calculus-based physics class - all male - with engineering majors - electrical, mechanical or civil engineering.

All of the students were in either their sophomore or junior years, and conceptual physics or calculusbased physics were the only physics courses that they had all taken in college. All but one of them had taken physics classes in high school. Half of the students were motivated by extra credit and half were attracted by a small cash payment. Each interview lasted for about 30-40 minutes.

In the beginning of each interview students were presented with four X-ray pictures - three medical ones - the hand, the skull and the breast and one nonmedical - an image of a bag screened by an airport security camera. They were also shown three or four other medical images that resulted from ultrasound, MRI and CAT scans and were asked what they could tell about them. Then our discussion went through various physics concepts that students eventually brought up in the conversation - light, waves, particles, spectrum, etc. The students were asked to compare Xrays to ultrasound and other imaging techniques, prompted to recall details from their personal experiences with X-rays and encouraged to use any information from other sources that they found relevant.

\section{Phase 2 - Fall 2005}

In phase 2 of our study we interviewed 10 junior and senior pre-med or other health-related majors who were currently taking algebra-based physics. We used a rigid, semi-structured (but otherwise very similar to
Phase 1) protocol, that also included a general selfreflective discussion where students were free to express any opinion about the topic of X-rays and medical imaging, their relevance in the pre-med Physics curriculum and their views on how they should be taught. We also added the question "How would you explain X-rays to a 12-year old child?" giving the students another chance to express their views in more simple and clear if not scientific terms. This last question allowed us to double-check their mental models about the phenomena.

\section{Phase 3 - Spring 2006}

Having accumulated extensive information on what to expect from our targeted pre-med audience, during Phase 3 , we focused more on the fact that the final results of our research would be the development of teaching materials. Therefore, we extended the interview process into two stages - one clinical and one teaching interview with each student. The first stage remained basically unchanged from Phase 2 (since it proved to be comprehensive enough and allowed comparison for reliability purposes).

Addressing the issue of the electromagnetic (light) nature of X-rays, their different ways of interaction with the materials of different properties (and also addressing geometrical issues that may arise during CAT scan image processing) we designed a small individual lab that used LEGO bricks.

We also have taken into account the learning cycle paradigm [7] - so the exploration, introduction of a concept and the application of a concept stages could be more or less clearly identified. This sequence was built around the "most convenient" concept - the blocking ability of a material. (Strictly speaking, this blocking ability should be separated into reflection, refraction and absorption; however for our imageprocessing purposes this elaboration was not necessary).

The students were presented with a "black box" which was built from semi-transparent LEGO bricks and were told that an object of an unknown shape was inside and that it is made out of the same semitransparent bricks as the walls. (There was no nondestructive way that they could see it directly.). Students were asked how they could determine the shape of this object. If students were unable to answer the question, a couple of scaffolding steps were provided, which included showing students the source of light (red LED, light-emitting diode) and the light detector (photovoltmeter).

To facilitate the task and the discussion another similar box with an object of a different shape inside was shown to students. In this case they were allowed 
to open it before making assumptions (the exploration stage finished here).

Subsequently the students were asked how they thought the readings of the photovoltmeter depended on the number of bricks through which the light had gone through. They were prompted to make a prediction about what would happen if we were to add more bricks one by one and measure the light that had gone through that sequence. This concluded the concept introduction stage.

Then the students made their final prediction about the result - what is inside the first box (the final application of the concept). They again engaged in a general discussion about the activities and their order and relevance in the pre-med labs followed.

Overall, five pre-med students, five other healthrelated majors and two engineering students were interviewed. One student who participated in Phase 2 took part only in the teaching interview (stage 2) this time.

\section{RESULTS \& DISCUSSION}

Many students felt confused when they were asked what else they could say about X-rays as they had almost never thought about the subject. They even declared that they did not know anything - although they often actually knew enough to answer our main questions. They just needed some encouragement, patience and scaffolding from the interviewer to invoke the transfer. Calculus-based physics students were more knowledgeable about the general topics of physics but that did not help them much to build up a coherent model of X-rays.

The pre-med students who were clinically interviewed during Phase 2 and 3 were much more interested in the dialog, felt that they should have known about it, and even very knowledgeable and assiduous students expressed this "constructive frustration" during the early stages of the interview. Then they openly and extensively talked about it in the self-reflective part of the interviews.

All of the pre-med students were enthusiastic about the Phase 3 teaching interview. Engineering majors were more reluctant while other pre-healthprofessionals could be described as having been moderately willing.

One of the most eager pre-med students responded: "I think it's really cool... interesting and... I mean... it's one of the most interesting physics kind of labs... kinds of things I ever done. It really gives the idea of what's going on..."

There was only one exception where a student thought that the proposed lab was somewhat "irrelevant" but even in that case the student liked the routine; she just did not believe that a physics lab could be likable: "I don't know whether it would be any better... I mean I liked this... you could really see more visually... we did really do stuff like this with $X$ rays."

Essentially our study revealed the following main themes:

1) Pre-med (and some other) students' ideas about X-rays can be described as models although these models are rarely consistent. Even for the students with greater knowledge (like pre-engineers) and greater interest (like pre-meds) these models are incoherent. Although in the interview process, through Socratic dialogue and careful leading, they often successfully tried to put together the pieces of knowledge transferred from their physics classes and combine them with other pieces of information. These models typically include the following components:

a) Almost all the interviewed students associate $X$ ray visibility of different objects with their density (12 out of 13 for the last phase) although not necessarily regular mass density, sometimes they mean something different - like concentration. However they sometimes mention other possible options: "They (darker regions on an X-ray picture) are dense... Or may be just because of the structure of it? Permeability, I guess... But I think it has more with the density." So these strong and stable intuitive associations can still be characterized as basic elements of reasoning (how things "simply happen”) so called phenomenological primitives (p-primes) [8]

b) Those students who successfully invoked and transferred their knowledge that X-rays are a part of "the spectrum" (12 out of 13) usually cannot recall whether they belong to the longer or shorter part of the wavelength spectrum (7 out of 13); they even tend to put them not randomly but rather mistakenly in the longer part of the spectrum, apparently making the association "longer - bigger - stronger.", here we observe the whole combination or chain of p-primes

The characteristic of wavelength comes into their mind much more easily and quickly than frequency and this fact affects their further conclusions a lot.

c) This previous association is coupled together with another important one - that X-rays are more damaging than most of the others so they have to be "stronger" and "bigger" in some relation.

d) When prompted to think about other wave characteristics of X-rays - like frequency - those students who chose longer wavelength for X-rays tended to change their opinion - now higher frequency is already associated with stronger, more dangerous waves including X-rays.

2) Students also easily recognize ultrasound (sonogram) pictures. They successfully transfer almost all of the sound properties to ultrasound, although how 
exactly ultrasound pictures are produced appears to be a mystery to them. They easily assume that taking ultrasound pictures isn't hazardous since it's used for looking at delicate unborn babies without any safety measures: "With $X$-rays I have to take a lot of precautions and you want to limit the exposure... With ultrasound I've never heard that... so I am thinking that it's OK'. Students understand that sound is more like a "vibration" and light is something different. They express this distinction using different terms such as radiation, photons, particles, perpendicular magnetic and electrical and transfer different "signature" features and concepts. Of course the usual particle-wave duality difficulties arise here and we often pursued them although this was not the main purpose of our research.

3) Students who can recall more details from the $\mathrm{X}$-ray procedures tend to associate X-rays with shadow-like processes since we are recording the light that passes (or doesn't pass) through our bones instead of the light that is reflected from them as we do in regular photography (their transfer of learning from personal experience overcomes others sources here): "Photography... is basically... when you are flashing... and getting... and it coming back to your camera... while this is still on the other side of the hand... that is going through... it's $X$-rays that make it through... and hit the film... and may it turn white."

However, when asked how they would explain $\mathrm{x}$ rays to a 12-year old they often just said that it was making a picture of you with rays that could penetrate through the skin - so again they appear to retreat to the photography analogy: "I would explain X-rays take a picture, but the flash on the camera can show through the skin... except for the bones... so it's like taking picture of the bones." Thus, their judgment appears to be very context-dependent.

\section{CONCLUSION AND FUTURE WORK}

During our study we found evidence that students transfer pieces of knowledge from very different sources such as their own X-ray experiences, previous Physics and other science courses and the mass media. This transfer results in mental models that are not necessary stable, consistent or coherent. However, these models are popular and persistent among pre-med students and should be taken into account when designing the $\mathrm{X}$-ray module for our Medical Physics curriculum and we have to build on them when planning new instructional materials. Students liked the proposed teaching activities, enthusiastically learned from them and we will continue develop them, taking into account the issues that arose in pre-med physics education in general and in our interviews series particularly.

\section{REFERENCES}

1. Nelson Christensen, Eur. J. Phys. 22 421-427, Medical physics: the perfect intermediate level physics class (2001)

2. Suzanne Amador, Biophysical Journal Vol. 66 June 2217-2221, Teaching Medical Physics to General Audiences (1994)

3. Dean Zollman, Modern Miracle Medical Machines: a course in contemporary physics for future physicians Proceedings of Groupe International de Recherche sur l'Enseignement de la Physique (GIREP), Lund, Sweden (2002)

4. N. Sanjay Rebello, Dean A. Zollman, Alicia R. Allbaugh, Paula V. Engelhardt, Kara E. Gray, Zdeslav Hrepic, Salomon F. Itza-Ortiz, Dynamic Transfer: A perspective from Physics Education Research in $\mathrm{J}$. Mestre (Ed.), Transfer of learning: Research and perspectives. Greenwich, CT: Information Age, 2002

5. Dean Zollman, NSF grant proposal: "Technology \& Model-Based Conceptual Assessment,” 1999

6. Jean Piaget. The Principles of Genetic Epistemology; Basic Books, New York, 1972

7. Robert Karplus, Science Curriculum Improvement Study: Teachers Handbook. Lawrence Hall of Science, Berkeley, CA, 1974

8. Andrea diSessa. Cognition and Instruction, 10, 105-225, Toward an epistemology of physics (1993) 